\title{
Case Report: A Predictable Technique to Establish Occlusal Contact in Extensive Direct Composite Resin Restorations: The DSO-Technique
}

\author{
NJM Opdam • JA Skupien • CM Kreulen \\ JJM Roeters • BAC Loomans • MC DNJM Huysmans
}

\section{Clinical Relevance}

This technique enables the dentist to restore teeth in occlusion with direct composites in a predictable and minimally invasive way.

\section{SUMMARY}

This paper describes the application technique of direct shaping by occlusion (DSO) for large composite restorations including the entire occlusal surface. For this technique, matrix bands and wedges are inserted without interference with antagonists in the desired occlusion. The final increment of soft-composite resin is shaped by letting the patient occlude on the uncured composite. Due to the nature of

*Niek JM Opdam, DDS, PhD, associate professor, Radboud Institute for Molecular Life Sciences, Department of Dentistry, Radboud university medical center, Nijmegen, The Netherlands Jovito A Skupien, DDS, MSc, PhD, adjunct professor, School of Dentistry, Franciscan University Center, Santa Maria, Brazil.

Bas AC Loomans, DDS, PhD, assistant professor, Radboud Institute for Molecular Life Sciences, Department of Dentistry, Radboud university medical center, Nijmegen, The Netherlands

Marie-Charlotte DNJM Huysmans, DDS, PhD, professor and head, Radboud Institute for Molecular Life Sciences, Department of Dentistry, Radboud university medical center, Nijmegen, The Netherlands the technique, special care has to be taken for moisture control and handling of contamination. The procedure, advantages, and limitations of the technique are discussed.

\section{PURPOSE}

Direct composite resin restorations have been shown to be suitable to provide a solution in a wide variety of indications with long-lasting results. ${ }^{1-6}$ Even in total rehabilitations of severely worn dentitions, direct composites have been shown to be successful ${ }^{7-9}$ on a

Joost JM Roeters, DDS, PhD, professor, Department of Restorative Dentistry, ACTA (Academic Centre for Dentistry), Amsterdam, The Netherlands

Cees M Kreulen, DDS, PhD, associate professor, Radboud Institute for Molecular Life Sciences, Department of Dentistry, Radboud university medical center, Nijmegen, The Netherlands

*Corresponding author: Philips van Leydenlaan 25, PO Box 9101, 6500 HB Nijmegen - The Netherlands; e-mail: niek. opdam@radboudumc.nl

DOI: $10.2341 / 13-112-\mathrm{T}$ 
medium to long term (5-10 years) and sometimes even preferred over indirect full-ceramic restorations. ${ }^{10}$ However, the direct restoration of teeth with extensive defects is a demanding procedure for most clinicians, especially when a new occlusion has to be established. Some dentists use moulds fabricated from a wax-up by the dental technician to apply the resin composite to the teeth. ${ }^{10,11}$ Recently, in this journal a technique was described to restore severely worn teeth with direct composite pairwise in order to establish interocclusal relationship. ${ }^{12}$ However, making proper restorations in the desired shape and in good occlusion is time-consuming and requires special skills from the dentist.

Frequently, indirect restorations are chosen in such a situation, ${ }^{13-16}$ but even when these indirect restorations are made in a minimally invasive way, ${ }^{16}$ costs are extensive and not affordable for most patients. Direct composite has the advantage of combining reasonable cost and proven longevity even in severely damaged dentitions. ${ }^{7,8}$ Moreover, it can be applied in a minimally invasive way offering possibilities for future restorative options in a dynamic way. ${ }^{11,12,17,18}$

The aim of this paper is to describe a technique facilitating occlusal rehabilitation on a mid-long term basis at limited costs. The technique was introduced in a Dutch language paper. ${ }^{19}$ Its clinical effectiveness has been established as restorations made according to this technique have been shown to have a favorable longevity. ${ }^{7}$

\section{DESCRIPTION OF THE TECHNIQUE}

The essential part of the technique is that to establish occlusion, the final increment of composite resin covering the occlusal surface is left uncured when the patient is asked to occlude and keep the teeth in occlusion, while the composite resin is cured from the buccal side (DSO = direct shaping by occlusion). By coating the antagonist with a thin layer of Vaseline, bonding and sticking of the composite will be avoided when cured. The technique facilitates the shaping of a restoration that includes the entire occlusal surface. In the following case presentations, the technique will be described in further detail.

\section{CASE 1}

A 41-year-old man with severe tooth wear was referred to the clinic of the dental school in Nijmegen. The anamnesis revealed that bruxism was the reason for the wear. The patient complained about the esthetic appearance related to the reduced size of his teeth. During the informed consent, it was decided to start a restorative treatment including a complete build-up of all anterior and posterior teeth in increased vertical dimension of occlusion (VDO). Furthermore, he was advised to wear a night guard for protection after the rehabilitation was completed.

Before starting the restorative treatment, individual stone casts were mounted in an articulator in maximal occlusion, and the desired VDO $(+5 \mathrm{~mm})$ was determined by the operator, carefully considering the necessary interocclusal space in posterior and anterior teeth. To transfer the new vertical dimension into the mouth, polyvinyl siloxane stops were made in the molar region of the casts (Figure 1A). The new VDO was checked in the mouth (Figure 1B).

In this case, the reconstruction started with direct composite resin build-ups of the mandibular anterior teeth, which is not relevant for the DSO technique as shown. Preparation of all involved teeth was limited to a bevel ground on the sharp edges of enamel, when present. In the cases where retention of the restoration could be at risk because available tooth substance is limited or applied heavy shear forces are expected, some resistance form can be created by grinding seats or grooves. When the mandibular anterior teeth were shaped and finished, attention was paid to have sufficient space left between the restored mandibular incisors and the unrestored maxillary anterior teeth with stops in situ in order to allow restoration of their palatal surfaces. After finishing and polishing the mandibular anterior teeth, a metal matrix band (Tofflemire) was inserted from the palatal side of a central maxillary incisor and secured with wooden wedges. Before placement of the matrix, the estimated height of the metal was adjusted with scissors. After placement, the matrix band was adjusted once more using a high-speed diamond bur. Finally, the matrix was checked in occlusion on the stops in increased VDO as there should be no interference between matrix and antagonists (Figure 1C). Although it was intentional that no rubber dam was applied, appropriate moisture control was achieved using cotton rolls, suction device, and proper matrixwedge placement while a dental nurse facilitated the procedure at chair side.

Applying a three-step etch-and-rinse adhesive (Clearfil Photo Bond, SA primer) according to the manufacturer's instructions started the restorative procedure. Then, a small amount of flowable composite was injected into the cervical area and left uncured to act as lubricant and to improve adaptation of the restorative material (snow-plough technique). ${ }^{20}$ This 


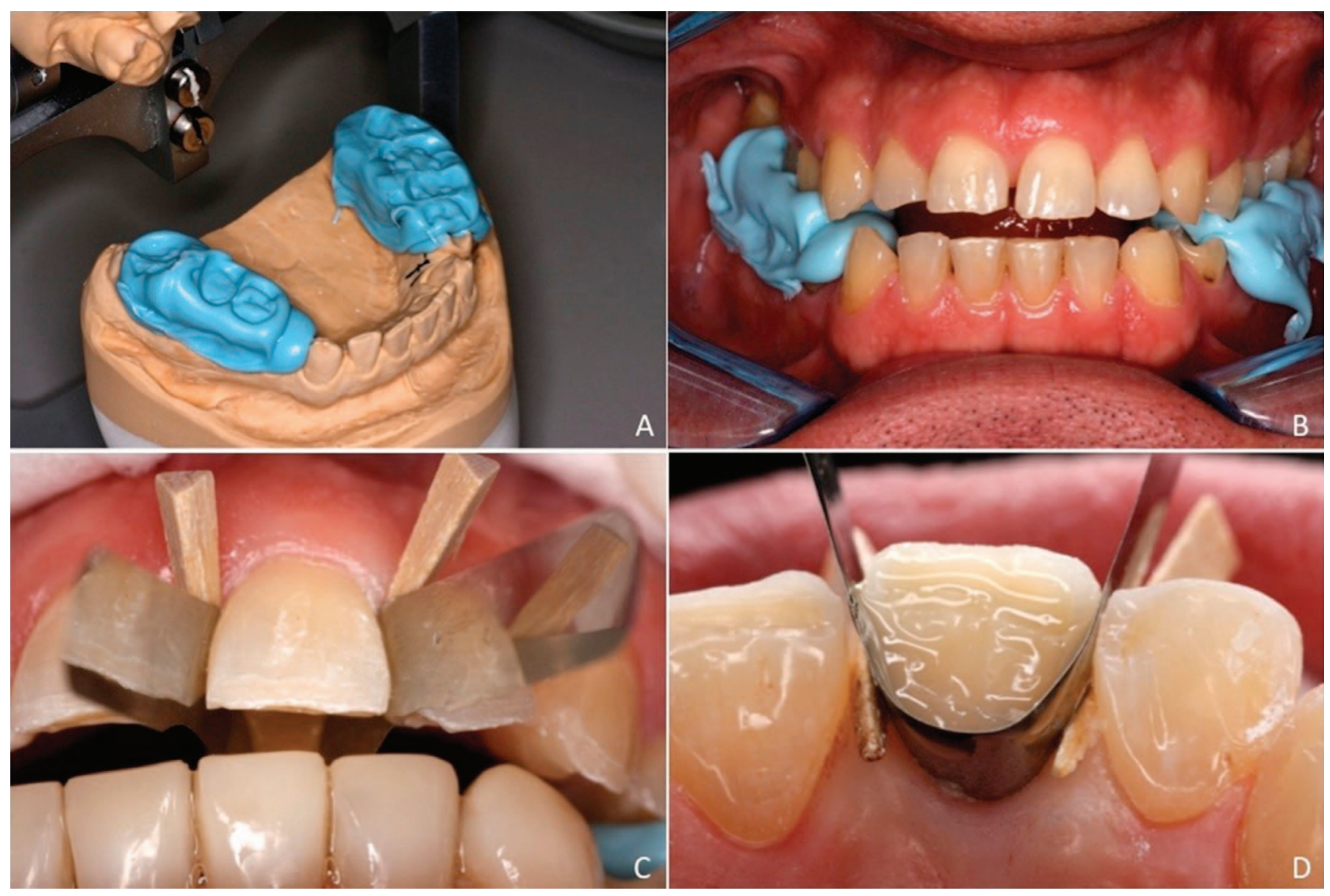

Figure 1. (A): Stops made on the mounted casts for transferring the desired VDO into the mouth. (B): Checking new VDO in the mouth. (C): Vestibular view of matrix band secured with wedges enabling full closure in the occlusal stops. (D): Palatal view of the tooth with matrix band and wedges after the application of the first layer of composite, which is cured normally. The correct placement of matrix and wedges will result in adequate moisture control.

was immediately followed by the first cervical layer of hybrid composite (Clearfil AP-X) that was applied and cured for 20 seconds, while care was taken that this layer was placed up to a level $1 \mathrm{~mm}$ below the matrix (Figure 1D). Subsequently, the final layer of the hybrid composite was inserted covering the entire surface inside the matrix. The thickness of this final layer was estimated to be sufficient for the antagonistic teeth to make a clear impression in the uncured composite. This layer was left uncured, while the antagonistic tooth was coated with a thin film of Vaseline. Hence, the suction device was removed, and the patient was asked to close the mouth into the stops and keep the teeth in occlusion (without biting with force) resulting in a direct shaping of the palatal surface by the antagonist (DSO). Then, with the jaws closed in the increased VDO stops, the composite was cured from the buccal side (Figure 2A). After curing for at least 40 seconds with a powerful curing-light unit $\left(>1000 \mathrm{mw} / \mathrm{cm}^{2}\right)$, the patient was asked to open the mouth, and the composite was additionally cured from the occlusal direction (20 seconds), and once more from the lingual side after removal of the metal matrix (20 seconds). In this case, the vestibular surface was subsequently restored with a direct veneer using an anterior composite (Empress Direct), resulting in the first tooth to be finished (before polishing) (Figure 2B). Figure 2C shows the shape of the finished antagonist contact area on the palatal surface. Note that occlusion was checked and all interferences in eccentric direction were removed before the second tooth was restored. Figure 2D shows the application of the matrix on the second tooth and the first layer of uncured flowable composite resin applied before injection of the hybrid material using the snow-plough technique. ${ }^{20}$ All maxillary anterior teeth were reconstructed in a similar way, while the posterior stops were kept in situ during closing in order to have identical maximal occlusion. Every tooth was adjusted in occlusion to 


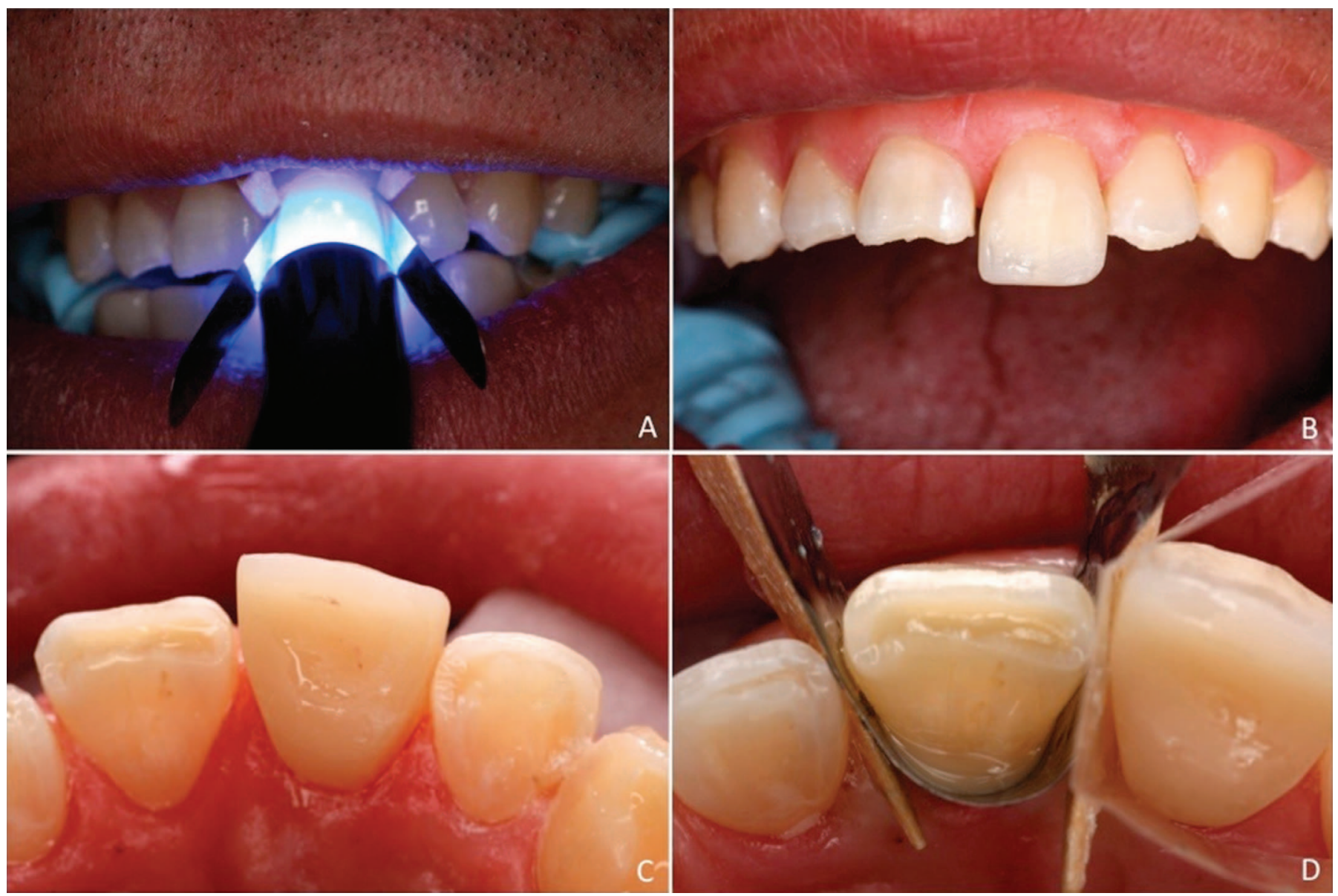

Figure 2. (A): Polymerization of the last layer of composite resin in occlusion (DSO). (B,C): First tooth finished before final polishing. Compare the size with the contra lateral incisor. (D): Matrix in situ and application of a thin layer of flowable composite in the cervical area of the second tooth (snow-plough technique).

the already built-up and finished teeth, and eccentric interferences were removed before treating the subsequent tooth. After removing interferences in lateral excursions on all incisors, the palatal surfaces of the canine teeth were adjusted so that a canine guidance was achieved.

Once the reconstruction of the maxillary anterior teeth was completed using the DSO technique, all teeth were polished. Figure $3 \mathrm{~A}$ through $\mathrm{C}$ shows the result with the anterior teeth restored in increased VDO. This was the end of the first treatment session (4 hours), and the patient was sent home for a week.

The next procedure to restore posterior teeth was as follows:

1. maxillary first premolars were built up in the estimated Curve of Spee in line with the maxillary canines (Figure 3D);

2. mandibular first premolars were restored using the DSO technique (Figure 3D);
3. mandibular posterior teeth were built up in the estimated right plane prior to restoring the maxillary antagonists (Figure $3 \mathrm{E}, \mathrm{F}$ ); and

4. maxillary second premolars and molars were restored using the DSO technique (Figure 4A through D).

For this part of the treatment, two treatment sessions of four hours were required. As with the anterior teeth, the occlusal surfaces were finished and interferences in lateral excursions were removed using fine-grit diamond burs, aiming at contact in occlusion and preserving canine guidance.

All teeth were polished and finished using abrasive discs and silicone tips (Figure 5A through C).

\section{CASE 2}

A 45-year-old woman was referred to the clinic with a bridge that repetitively had been dislodged. At the time the patient was seen, the bridge had been 


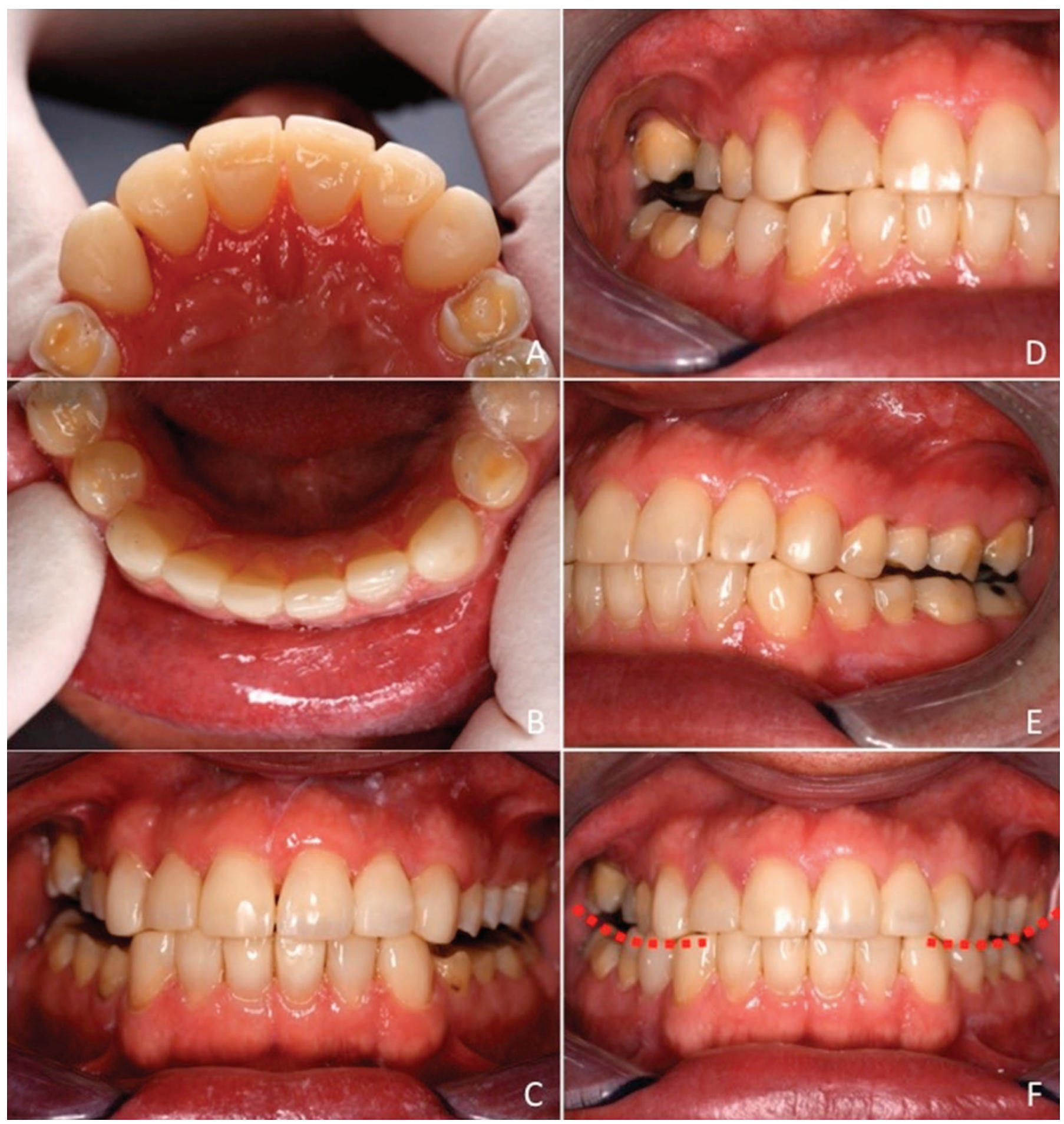

Figure 3. (A): Maxillary anterior teeth. (B): Mandibular anterior teeth. (C): First session completed. All anterior teeth restored, allowing reduced but stabilized occlusion including canine guidance. (D): Second session started with the first premolars being restored. Right arch. (E): Left arch, including mandibular posterior teeth restored. (F): Mandibular teeth are restored according to the Curve of Spee.

recemented three months earlier, and again the premolar-abutment tooth showed a dislodged crown (Figure 6A through C). The etiology was identified as bruxism, mainly by clenching the teeth. It was decided to remove the mesial part of the bridge and replace it with a direct composite crown to see if this solution would function on the mid-long term basis. It was agreed to decide later whether a new crown 


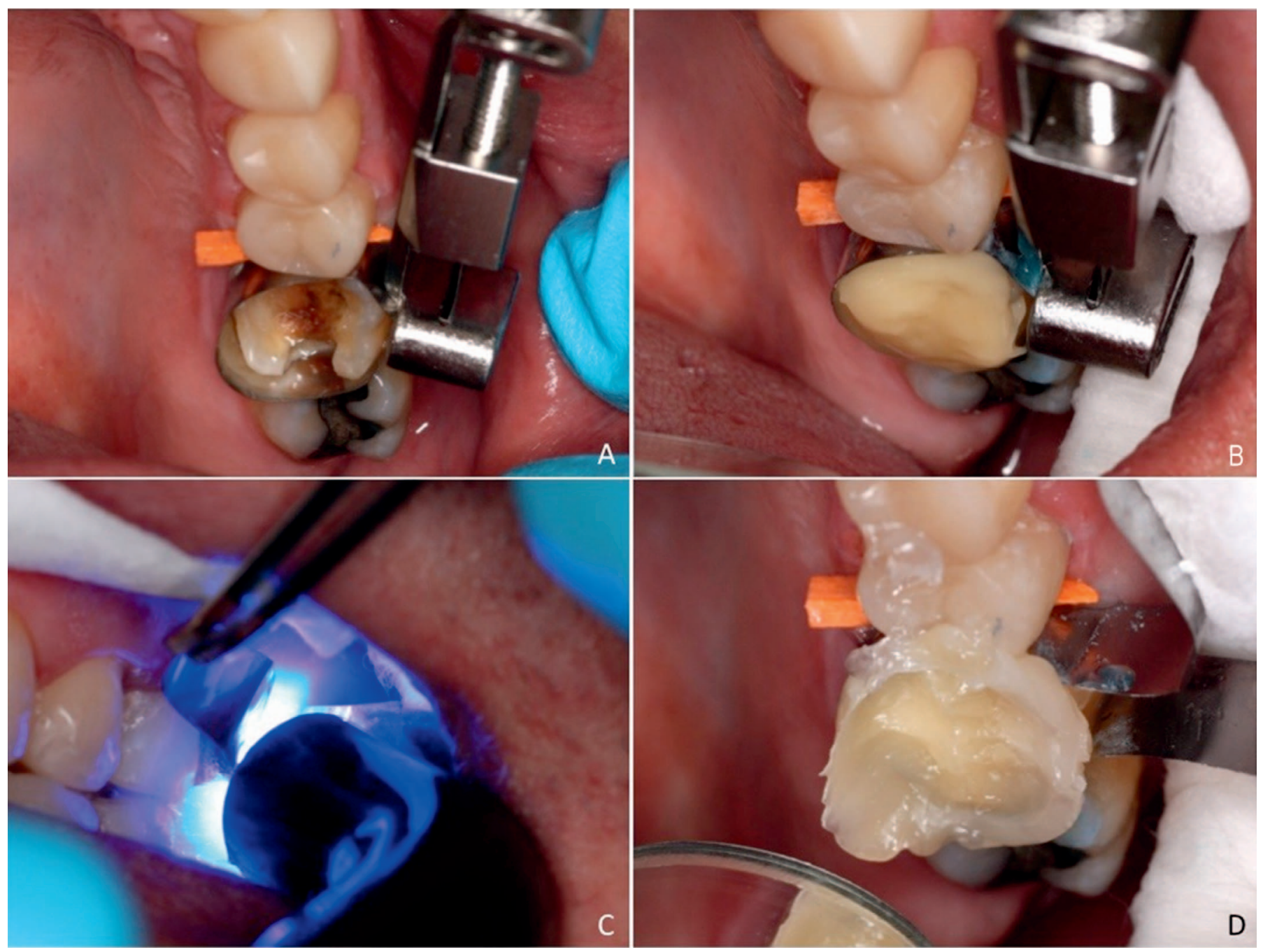

Figure 4. (A): In the first molar, the existing amalgam restoration was removed, a matrix band placed using a Tofflemire device and secured with wedges. It was verified that the patient was able to close in the desired VDO without interference. (B): The final layer of composite resin inserted on the occlusal surface left uncured. It is important to have excess of material to achieve an imprint of the opposing tooth. (C): Polymerization of the material after closing in occlusion (DSO technique). The Tofflemire device has been removed and the matrix-band was "opened" to have better access for the curing light. (D): First maxillary molar immediately after opening the mouth, after the final curing.

had to be made and if the diastema had to be closed again, probably with an implant-supported crown. After informed consent, the mesial part of the bridge was removed (Figure 6D). The core proved to be suitable for a direct composite build-up. This was done using the DSO technique.

First, moisture control was achieved using cotton rolls placed buccally and lingually and inserting a suction device. Due to the healthy condition of the gingiva and the absence of inflamed tissue prone to bleeding, it was decided to do the adhesive procedure without a matrix placed. After the adhesive procedure using a two-step self-etching adhesive (Clearfil SE Bond), a partial metal matrix band was placed, using the operator's finger to control the position on the lingual surface in the gingival sulcus (Figure 7A). Subsequently, a layer of hybrid composite was inserted in the matrix, shaped, and cured (Figure 7B). Then, the matrix was removed and the buccal cervical surface was built up in the same way (Figure 7C). Subsequently, another partial metal matrix band was placed mesially and secured with a wedge and a separation ring to establish proximal contact (Figure 8A,B). When the mesial, buccal, and lingual surfaces were built up and cured properly, the occlusion was checked after removal of cotton rolls and suction device. It showed that the first build-up interfered in maximal occlusion resulting in the composite to be reduced with a high-speed diamond bur (Figure 8C). 

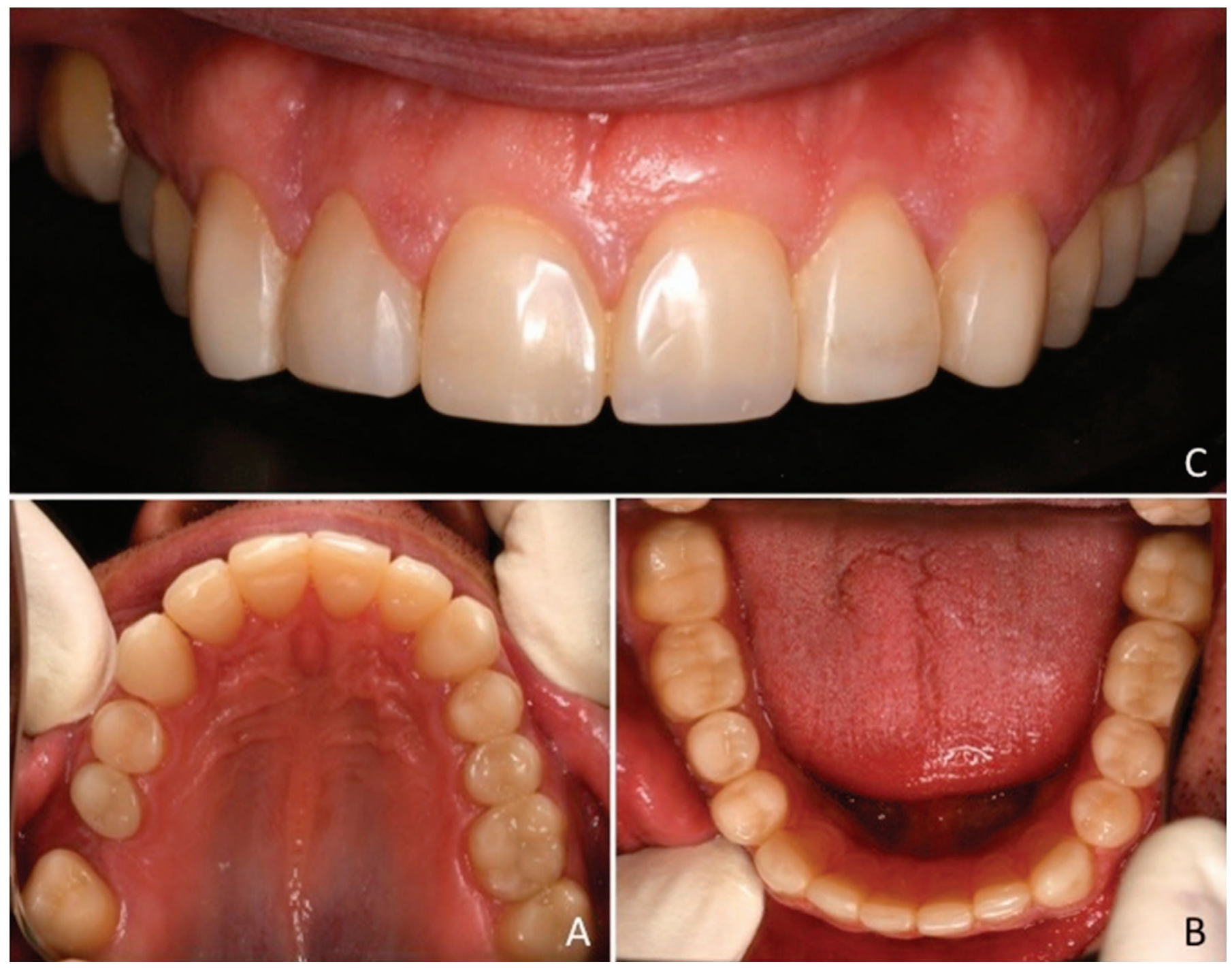

Figure 5. (A) Maxillary jaw: restorations finished and polished. (B): Mandibular jaw: restorations finished and polished. (C): Maxillary anterior teeth after polishing and finishing the restorations.

Hence, a Tofflemire matrix band was applied. A high-speed diamond bur was used to reduce the metal matrix so that no interference with antagonistic teeth remained when closing the mouth in maximal occlusion. No separation ring was required at this point because the proximal contact was already constructed in the first step. Then again, moisture control was achieved by cotton roll and suction device isolation. The adhesive procedure was repeated by applying phosphoric acid, rinsing, and applying the adhesive. Finally, the occlusal part of the restoration was restored using the DSO technique (Figure 9A through D).

Figure 10 (A through D) shows the case completed and follow-up after six months.

\section{List of Materials}

- Clearfil AP-X (Kuraray, Osaka, Japan);

- Clearfil Majesty Flow (Kuraray, Osaka, Japan);

- IPS Empress Direct (Ivoclar Vivadent, Schaan, Liechtenstein);

- Clearfil Photo Bond, SA Primer, (Kuraray, Osaka, Japan);

- Phosphoric acid 35\% (DMG, Hamburg, Germany);

- Clearfil SE Bond (Kuraray, Osaka, Japan);

- Sof-Lex pop-on polishing discs (3M ESPE, St Paul, MN, USA);

- Silicone tips (Cosmedent, Chicago, IL, USA);

- Tofflemire matrix system and bands (Henry Schein, Melville, NY, USA); and

- Partial matrix bands (Palodent, Dentsply, Konstanz, Germany) 

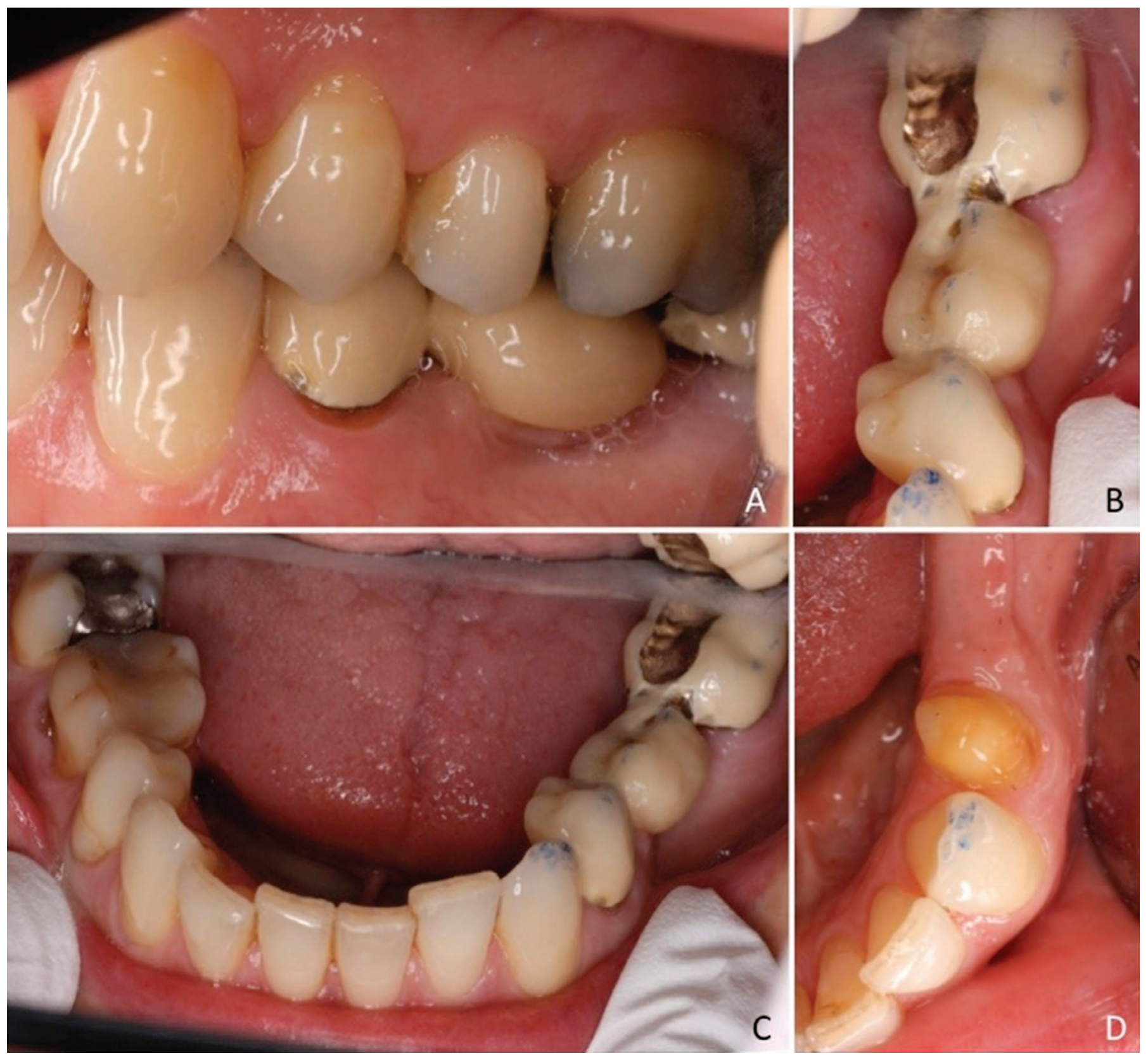

Figure 6. $(A, B, C)$ : $A$ bridge from tooth 35 to 37 has failed repetitively by dislodgment of the mesial abutment tooth. (D): Core visualization of the premolar after the mesial abutment crown and pontic were removed.

\section{Potential Problems}

The presented technique is a relatively inexpensive alternative for placing extensive restorations in occlusion and can be applied in several situations, including cases that require increased vertical dimension. Clinical long-term results for restorations placed in increased VDO using this technique are limited to one retrospective analysis showing good results on a mid-long term basis. ${ }^{7}$ More evidence on the survival of resin composite restorations when patients with severe tooth wear are restored in increased VDO is limited to two other studies, ${ }^{8,21}$ including one that shows unfavorable outcome after three years when a microfilled composite was used. ${ }^{21}$ However, clinical studies on restorative treatment of patients in increased VDO with indirect techniques are absent, and evidence is limited to case reports. ${ }^{10,16,22}$ Although some clinical evidence is present that this technique is working in clinical dentistry, long-term results are still questionable, and some concerns that might arise must be addressed. 

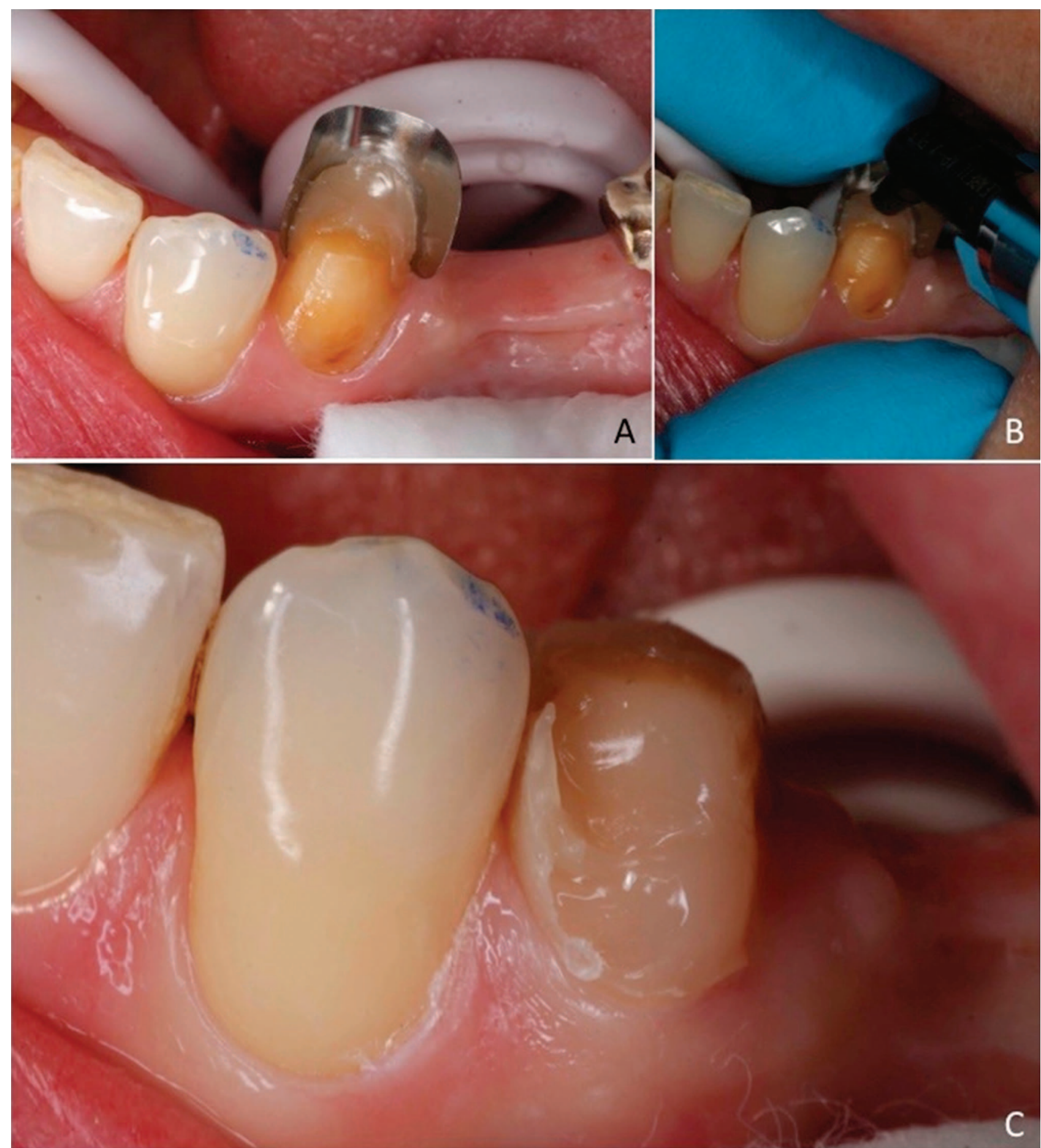

Figure 7. (A): Adaptation of the partial matrix band in the gingival sulcus controlled by finger pressure. Composite resin being inserted from the preloaded tip. (B): First layer of composite cured. (C): Premolar after applying the buccal portion of composite.

\section{Moisture Control}

For the DSO technique, moisture control cannot be achieved by placing a rubber dam because this would prevent the patient from biting in occlusion. The authors are aware of the often-stated recommenda- tion to use a rubber dam when an adhesive restoration is placed, but want to make a statement for the applied moisture control using cotton rolls, also based on the available scientific evidence. There are some clinical studies showing that regarding 


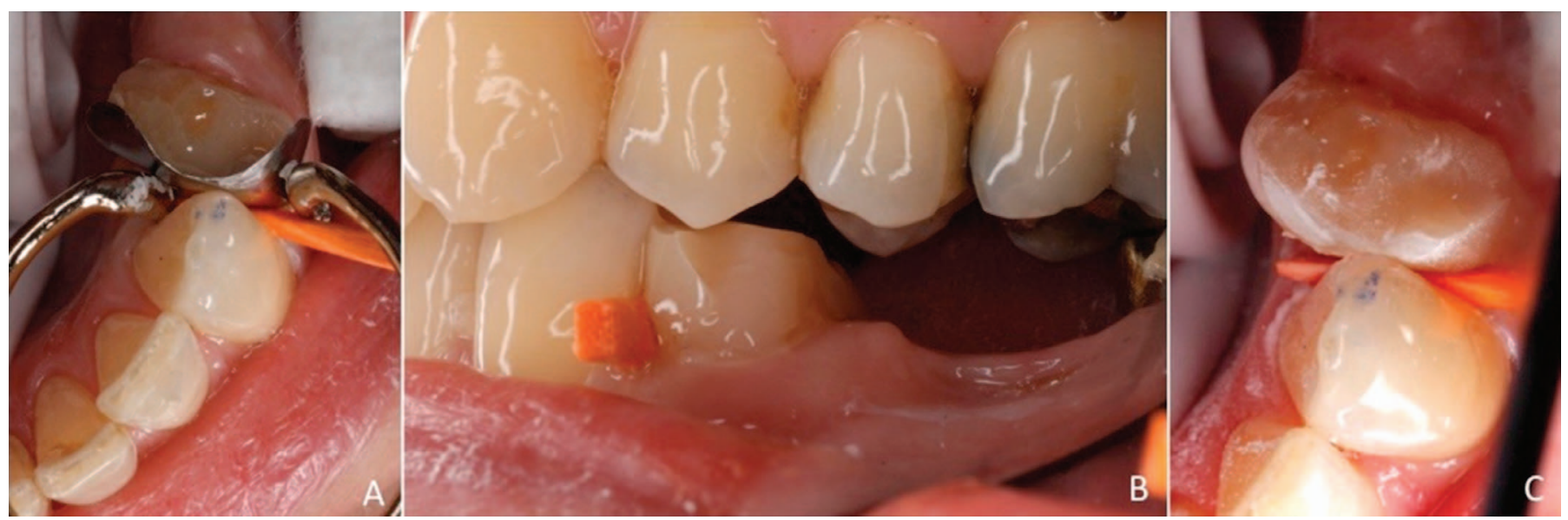

Figure 8. (A) Band, ring, and wedge located mesially. (B,C): Vestibular and occlusal view after reducing the composite to remove interferences in occlusion.

sealant retention and adhesive bridge retention, rubber dam plays a positive role in success rates, ${ }^{23,24}$ but a 10-year study comparing clinical survival of posterior composite restorations placed either with cotton rolls or with a rubber dam isolation method showed no differences in clinical outcome. ${ }^{25}$ Moreover, several long-term clinical survival studies on posterior composite restorations placed without a rubber dam show good results. ${ }^{4,26,27}$ Even when cervical outlines of class II restorations placed without a rubber dam extended below the dentin enamel junction, ending in dentin, no increase in secondary caries rates could be observed compared to restorations ending proximally in enamel. ${ }^{28}$ This indicates that the placement of a rubber dam is not mandatory to have good results when placing posterior composite restorations.

However, with the DSO technique, patients are asked to articulate into uncured composite, which may compromise the quality of the restoration. The authors consider the following prerequisites as most important for a successful DSO technique.

1. Avoid, when possible, building up mandibular molars with this technique, as these teeth are the most difficult for maintaining moisture control. Moreover, in normal class I relation, maxillary molar teeth offer better opportunities to place the matrix and matrix device without interference with antagonists, and a better access for the curing light is possible.

2. Keep the period of closing in occlusion and possible moisture contamination as short as possible: remove suction when Vaseline is applied and the last uncured layer of composite is in shape. This means that when saliva is contami- nating the area, it only comes in contact with uncured composite that is pressed in the palatal direction due to the closing in occlusion, and this material has to be removed anyway during shaping and finishing. Therefore, when properly applied, contamination will not compromise the result.

3. Whenever contamination occurs with saliva, it is recommended to repeat the adhesive procedure $^{29,30}$ after reestablishing moisture control. When, after the DSO technique, additional layers of composite should be applied, which is not normal but may be the case, we recommend removal of composite contaminated with Vaseline with a high-speed bur and repeating the adhesive procedure after reestablishing moisture control. So when applied properly, moisture control is no problem with the DSO technique, though the authors admit that, especially with lack of specific skills, it can be a potential weakness.

\section{Restoring in Increased VDO}

Although often a splint is recommended to test the increased $\mathrm{VDO},{ }^{16}$ a recent review was not conclusive on the necessity of wearing a splint in advance when increased VDO was included in the treatment plan. ${ }^{31}$ That review paper concluded that restoration in increased vertical dimension can be done safely up to $5 \mathrm{~mm}$. Moreover, the increased vertical dimension as applied in direct composite offers possibilities for adjustment of the occlusion; therefore, we are not recommending to use a splint in advance for these direct build-ups and, as the clinical report shows, no problems with the adjusted VDO were found. ${ }^{7}$ The method of positioning the occlusion by means of the stops offers the possibility to mount casts in central 


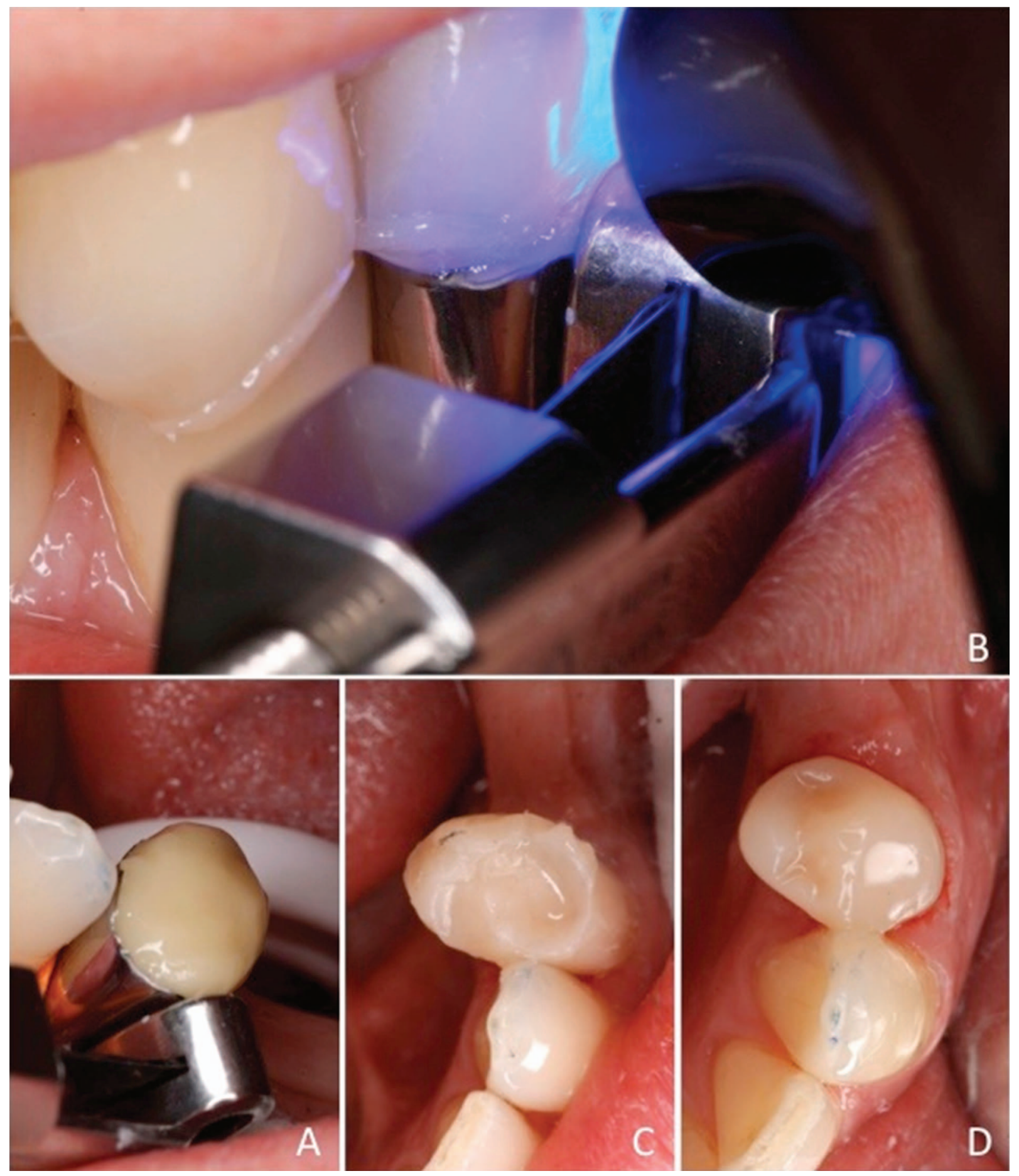

Figure 9. (A): Final layer of uncured composite resin. (B): Polymerization with Tofflemire in position and the mouth closed. Note the Vaseline applied on the antagonistic tooth. (C): Occlusal view immediately after the final polymerization. (D): Restoration finished and polished.

occlusion as well as central relation, and guides the patient's jaw in a reproducible position every time when closing the mouth. After opening the occlusion, many interferences still exist when patients make excentric movements or between central relation and central occlusion that need careful adjustment. It is important to do these adjustments after every new tooth is built up, so that before starting to build up a next tooth, central occlusion and lateral excursions are well established.

Moreover, as direct composite is used to create increased vertical dimension, this can be considered 


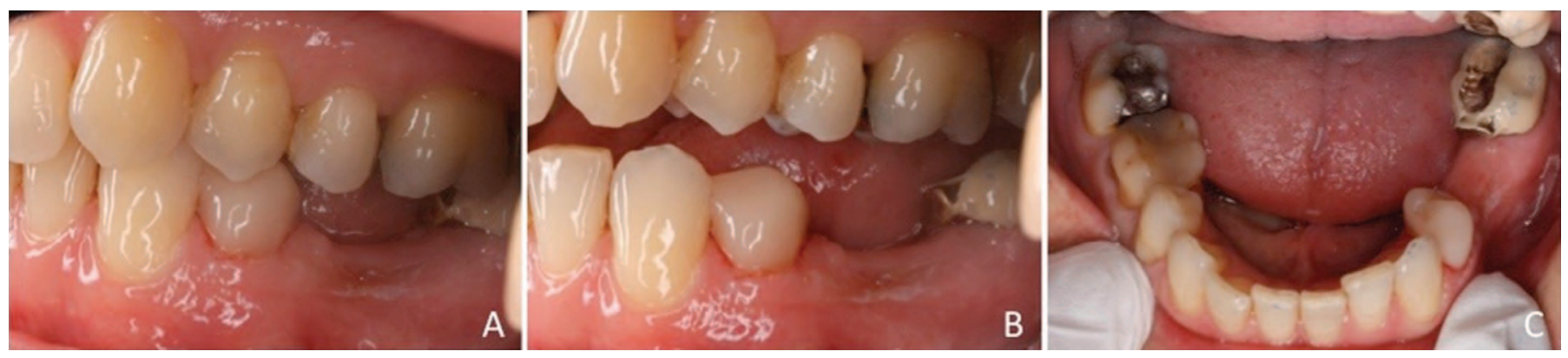

Figure 10. (A): Case completed. Vestibular view during occlusion. (B): Vestibular view during opening mouth. (C): Occlusal view after the conclusion of the case. (D): Six-month follow-up.

as "adjustable" if vertical dimension apparently is too high after a certain time. However, within the considerable experience that we have with the technique, we never have had a case where, after building up the occlusion, the vertical dimension had to be decreased in a following session due to complaints from the patient.

\section{SUMMARY OF ADVANTAGES AND DISADVAN- TAGES (OR LIMITATIONS)}

The DSO technique offers a possibility to restore teeth with direct composite in occlusion, especially in clinically complicated cases like replacing existing crowns or rehabilitations in increased vertical dimension. It is a relatively inexpensive technique that offers also the possibility to make mid-long term temporary restorations when large rehabilitations are to be done and new occlusal situations have to be tested. Especially for the restoration of severe tooth wear cases, the technique has proven itself as successful on a mid-long term basis.

However, the technique demands certain experience and skills of the operator as anatomic form and occlusal patterns are made manually. Moreover, certain skills of the dental team in moisture control are necessary as the technique is not possible with a rubber dam in place. For less experienced dentists, wax-up models and moulds ${ }^{11}$ may offer alternative options to do these extensive cases.

\section{CONCLUSION}

This newly presented technique can be a good choice to restore teeth with extensive tissue loss at relatively low cost. The technique has been proven successful for the treatment of severe tooth wear in increased VDO and is minimally invasive. It can also be used to make direct full coverage restorations in occlusion. Therefore, it can be a good option to restore teeth on the short- and middle long-term.

\section{Conflict of Interest}

The authors of this manuscript certify that they have no proprietary, financial, or other personal interest of any nature or kind in any product, service, and/or company that is presented in this article.

(Accepted 23 August 2013)

\section{REFERENCES}

1. Heintze SD, \& Rousson V (2012) Clinical effectiveness of direct class II restorations-A meta-analysis Journal of Adhesive Dentistry 14(5) 407-431.

2. Da Rosa Rodolpho PA, Donassollo TA, Cenci MS, Loguércio AD, Moraes RR, Bronkhorst EM, Opdam NJ, \& Demarco FF (2011) 22-Year clinical evaluation of the performance of two posterior composites with different filler characteristics Dental Materials 27(10) 955-963.

3. van de Sande FH, Opdam NJ, Da Rosa Rodolpho PA, Correa MB, Demarco FF, \& Cenci MS (2013) Patient risk factors' influence on survival of posterior composites Journal of Dental Research 92(7 Supplement) 78S-83S.

4. Opdam NJ, Bronkhorst EM, Loomans BA, \& Huysmans MC (2010) 12-year survival of composite vs. amalgam restorations Journal of Dental Research 89(10) 1063-1067.

5. Opdam NJ, Bronkhorst EM, Loomans BA, \& Huysmans MC (2012) Longevity of repaired restorations: A practice based study Journal of Dentistry 40(10) 829-835.

6. van Dijken JW (2010) Durability of resin composite restorations in high C-factor cavities: A 12-year followup Journal of Dentistry 38(6) 469-474.

7. Hamburger JT, Opdam NJ, Bronkhorst EM, Kreulen CM, Roeters JJ, \& Huysmans MC (2011) Clinical performance of direct composite restorations for treatment of severe tooth wear Journal of Adhesive Dentistry 13(6) 585-593.

8. Attin T, Filli T, Imfeld C, \& Schmidlin PR (2012) Composite vertical bite reconstructions in eroded dentitions after 5.5 years: A case series Journal of Oral Rehabilitation 39(1) 73-79.

9. Poyser NJ, Briggs PF, Chana HS, Kelleher MG, Porter RW, \& Patel MM (2007) The evaluation of direct composite restorations for the worn mandibular anterior dentition-Clinical performance and patient satisfaction Journal of Oral Rehabilitation 34(5) 361-376. 
10. Vailati F, Vaglio G, \& Belser UC (2012) Full-mouth minimally invasive adhesive rehabilitation to treat severe dental erosion: A case report Journal of Adhesive Dentistry 14(1) 83-92.

11. Schmidlin PR, Filli T, Imfeld C, Tepper S, \& Attin T (2009) Three-year evaluation of posterior vertical bite reconstruction using direct resin composite-A case series Operative Dentistry 34(1) 102-108.

12. Reston EG, Corba VD, Broliato G, Saldini BP, \& Stefanello Busato AL (2012) Minimally invasive intervention in a case of a noncarious lesion and severe loss of tooth structure Operative Dentistry 37(3) 324-328.

13. Brackett MG, Kious AR, \& Brackett WW (2009) Minimally retentive gold onlays: A six-year case report Operative Dentistry 34(3) 352-355.

14. Robinson FG, \& Haubenreich JE (2006) Oral rehabilitation of a young adult with hypoplastic amelogenesis imperfecta: A clinical report Journal of Prosthetic Dentistry 95(1) 10-13.

15. Sari T, \& Usumez A (2003) Restoring function and esthetics in a patient with amelogenesis imperfecta: A clinical report Journal of Prosthetic Dentistry 90(6) $522-525$.

16. Güth JF, Almeida E Silva JS, Ramberger M, Beuer F, \& Edelhoff D (2012) Treatment concept with CAD/CAMfabricated high-density polymer temporary restorations Journal of Esthetic and Restorative Dentistry 24(5) 310-318.

17. Wolff D, Kraus T, Schach C, Pritsch M, Mente J, Staehle HJ, \& Ding P (2010) Recontouring teeth and closing diastemas with direct composite buildups: A clinical evaluation of survival and quality parameters Journal of Dentistry 38(12) 1001-1009.

18. Alonso V, \& Caserio M (2012) A clinical study of direct composite full-coverage crowns: Long-term results Operative Dentistry 37(4) 432-441.

19. Roeters J, \& Stel M (2000) Adhesive techniques in the treatment of tooth wear due to bruxism [in Dutch] Nederlands Tijdschrift voor Tandheelkunde 107(7) 308-311.

20. Opdam NJ, Roeters JJ, de Boer T, Pesschier D, \& Bronkhorst E (2003) Voids and porosities in class I micropreparations filled with various resin composites Operative Dentistry 28(1) 9-14.
21. Bartlett D, \& Sundaram G (2006) An up to 3-year randomized clinical study comparing indirect and direct resin composites used to restore worn posterior teeth International Journal of Prosthodontics 19(6) 613-617.

22. Ardu S, Duc O, Krejci I, \& Perroud R (2013) Amelogenesis imperfecta: A conservative and progressive adhesive treatment concept Operative Dentistry 38(3) 235-241.

23. Ganss C, Klimek J, \& Gleim A (1999) One year clinical evaluation of the retention and quality of two fluoride releasing sealants Clinical Oral Investigations 3(4) 188-193.

24. Creugers NH, De Kanter RJ, Verzijden CW, \& Van't Hof MA (1998) Risk factors and multiple failures in posterior resin-bonded bridges in a 5-year multi-practice clinical trial Journal of Dentistry 26(5-6) 397-402.

25. Raskin A, Setcos JC, Vreven J, \& Wilson NH (2000) Influence of the isolation method on the 10-year clinical behaviour of posterior resin composite restorations Clinical Oral Investigations 4(3) 148-152.

26. Pallesen U, van Dijken JW, Halken J, Hallonsten AL, \& Höigaard R (2013) Longevity of posterior resin composite restorations in permanent teeth in Public Dental Health Service: A prospective 8 years follow up Journal of Dentistry 41(4) 297-306.

27. Pallesen U, \& Qvist V (2003) Composite resin fillings and inlays. An 11-year evaluation Clinical Oral Investigations 7(2) 71-79.

28. Kuper NK, Opdam NJ, Bronkhorst EM, \& Huysmans MC (2012) The influence of approximal restoration extension on the development of secondary caries Journal of Dentistry 40(3) 241-247.

29. Sheikh H, Heymann HO, Swift EJ Jr, Ziemiecki TL, \& Ritter AV (2010) Effect of saliva contamination and cleansing solutions on the bond strengths of self-etch adhesives to dentin Journal of Esthetic and Restorative Dentistry 22(6) 402-410.

30. Jiang Q, Pan H, Liang B, Fu B, \& Hannig M (2010) Effect of saliva contamination and decontamination on bovine enamel bond strength of four self-etching adhesives Operative Dentistry 35(2) 194-202.

31. Abduo J, \& Lyons K (2012) Clinical considerations for increasing occlusal vertical dimension: A review Australian Dental Journal 57(1) 2-10. 